\title{
Hall Effects on MHD Couette Flow in Rotating System
}

\author{
S. Das \\ Department of Mathematics, \\ University of Gour Banga, \\ Malda 731 103, India
}

\author{
B. C. Sarkar \\ Department of Applied \\ Mathematics, Vidyasagar \\ University, Midnapore 721 102, \\ India
}

\author{
R. N. Jana \\ Department of Applied \\ Mathematics, Vidyasagar \\ University, Midnapore 721 102, \\ India
}

\begin{abstract}
Hall effects on the MHD Couette flow between two infinite horizontal parallel porous plates in a rotating system under the boundary layer approximations have been studied. One of the plate is held at rest and the other one moves with uniform velocity. An exact solution of governing equation has obtained in closed form. Asymptotic behavior of the solution has analyzed for large values of magnetic parameter, rotation parameter and Reynolds number. It is observed that a thin boundary layer is formed near the stationary plate for large values of the rotation parameter, magnetic parameter and Reynolds number. The thickness of these boundary layers increases with increase in Hall parameter. The heat transfer characteristic has also discussed on taking viscous and Joule dissipations into account. It is found that an increase in Hall parameter, the temperature in flow field increases.
\end{abstract}

Keywords: Hall effects, MHD Couette, magnetic parameter, Hall parameter, rotation parameter, Reynolds number, Joule dissipations, heat transfer and boundary layer

\section{INTRODUCTION}

Magnetohydrodynamics is currently undergoing a period of great enlargement and differentiation of subject matter. The interest in these new problems generates from their importance in liquid metals, electrolytes and ionized gases. The mechanism of conduction in ionized gases in the presence of a strong magnetic field is different from that in a metallic substance. The electric current in ionized gases is generally carried by electrons which undergo successive collisions with other charged or neutral particles. In the ionized gases, the current is not proportional to the applied potential except when the electric field is very weak. However, in the presence of strong electric field, the electrical conductivity is affected by the magnetic field. Consequently, the conductivity parallel to the electric field is reduced. Hence, the current is reduced in the direction normal to both electric and magnetic fields. This phenomenon is known as the Hall effect. Due to this Hall current the electrical conductivity of the fluid becomes anisotropic and this causes secondary flow in magnetohydrodynamic primary flows. Hall current is of great importance in many astrophysical problems, Hall accelerator and flight MHD as well as flows of plasma in a MHD power generator. The study of the interaction of the Coriolis force with the electromagnetic force is also important. In particular, rotating MHD flows with heat transfer is one of the important current topics because of its applications in thermofluid transport modeling in magnetic geosystems, meteorology, turbo machinery, solidification process in metallurgy and in some astrophysical problems. It is generally thought that the existence of the geomagnetic field is due to finite amplitude instability of the Earth's core. Since most cosmic bodies are rotators, the study of rotating electrically conducting fluid is essential in understanding better the magnetohydrodynamics of the interiors of the Earth and other planets. It has motivated a number of studies on hydromagnetic rotating systems which can provide explanations for the observed variations in the geomagnetic field. Hall effects on hydromagnetic Couette flow and heat transfer has been studied by Gupta [1]. MHD Couette flow and heat transfer in a rotating system have been studied by Jana et al. [2]. Soundalgekar et al. [3] have studied the Hall effects on generalized MHD Couette flow with heat transfer. Hall effects on MHD plasma Couette flow in a rotating environment have been studied by Ghosh et al.[4]. Ghosh [5] has studied the effects of Hall current on MHD Couette flow in a rotating system with arbitrary magnetic field. The unsteady Couette flow of an electrically conducting fluid between two parallel plates with Hall effects has been investigated by Jana and Datta [6]. Kanch and Jana [7] have studied Hall effect on unsteady Couette flow under boundary layer approximations.

In the present paper we have studied the effects of Hall current on the MHD Couette flow when one of the plate moving with uniform velocity and the other one held at rest under boundary layer approximations. Closed form solution has obtained for the velocity field. Asymptotic behavior has also studied for large values of rotation parameter, magnetic parameter and Reynolds number. It is found that for large magnetic parameter the thickness of the boundary layer near the plate $\eta=0$ decreases with increase in Hall parameter $m$. On the other hand, for large rotation parameter as well as for large Reynolds number the boundary layer thicknesses increase with increase in Hall parameter.

\section{MATHEMATICAL FORMULATION AND ITS SOLUTION}

Consider the viscous incompressible electrically conducting fluid bounded by two infinite horizontal parallel porous plates separated by a distance $d$. Choose a cartesian co-ordinate system with $x$-axis along the lower stationary plate in the direction of the flow, the $y$-axis is normal to the plates and the $z$-axis perpendicular to $x y$-plane. The upper plate moves with a uniform velocity $U$ in the $x$-direction. The plates and the fluid are in a state of rigid body rotation with uniform angular velocity $\Omega$ about the $y$-axis. A uniform magnetic field $B_{0}$ is applied perpendicular to the plates. The velocity components are $(u, v, w)$ relative to a frame of reference rotating with the fluid. Since the plates are infinitely long, all physical variables, except pressure, depend on $y$ only. The equation of continuity then 
gives $v=-v_{0}$ everywhere in the fluid where $v_{0}$ is the suction velocity at the plates.

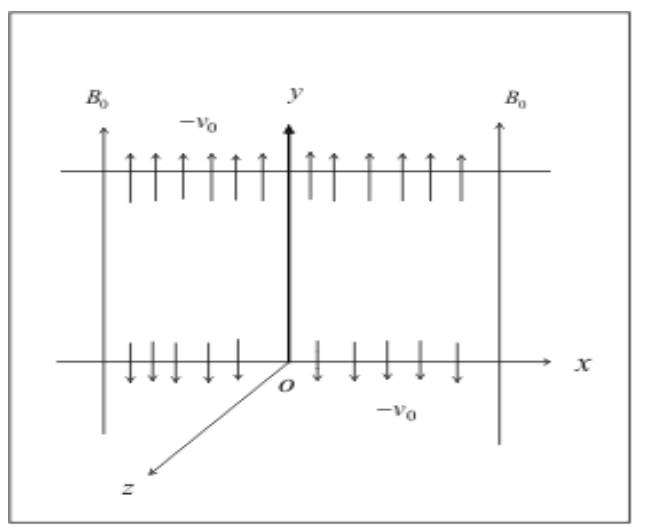

\section{Figure 1. Geometry of the problem}

Neglecting ion-slip and thermoelectric effects, the generalized Ohm's law for partially ionized gas is [see Cowling[8]]

$$
\vec{j}+\frac{\omega_{e} \tau_{e}}{B_{0}}(\vec{j} \times \vec{B})=\sigma(\vec{E}+\vec{q} \times \vec{B}),
$$

where $\vec{B}, \vec{E}, \vec{q}, \vec{j}, \sigma, \omega_{e}$ and $\tau_{e}$ are respectively, the magnetic field vector, the electric field vector, the fluid velocity vector, the current density vector, the conductivity of the fluid, the cyclotron frequency and the electron collision time.

We shall assume that the magnetic Reynolds number for the flow is small so that the induced magnetic field can be neglected. This assumption is justified since the magnetic Reynolds number is generally very small for partially ionized gases. The solenoidal relation $\nabla \cdot \vec{B}=0$ for the magnetic field gives $B_{z}=B_{0}=$ constant everywhere in the fluid where $\vec{B} \equiv\left(B_{x}, B_{y}, B_{z}\right)$. The equation of the conservation of the charge $\nabla \cdot \vec{j}=0$ gives $j_{z}=$ constant. This constant is zero since $j_{z}=0$ at each plates which is electrically non-conducting. Thus $j_{z}=0$ everywhere in the flow. Since the induced magnetic field is neglected, the Maxwell's equation $\nabla \times \vec{E}=-\frac{\partial \vec{B}}{\partial t}$ becomes $\nabla \times \vec{E}=\overrightarrow{0}$ which gives $\frac{\partial E_{x}}{\partial z}=0$ and $\frac{\partial E_{y}}{\partial z}=0$. This implies that $E_{x}=$ constant and $E_{y}=$ constant everywhere in the flow.

In view of the above assumption and on taking $E_{x}=E_{y}=0$, equation (1) gives

$$
\begin{aligned}
& j_{x}-m j_{z}=-\sigma B_{0} w, \\
& j_{z}-m j_{x}=\sigma B_{0} u,
\end{aligned}
$$

where $m=\omega_{e} \tau_{e}$ is the Hall parameter.

Solving for $j_{x}$ and $j_{z}$, we get

$$
\begin{aligned}
& j_{x}=\frac{\sigma B_{0}}{1+m^{2}}(m u-w), \\
& j_{z}=\frac{\sigma B_{0}}{1+m^{2}}(u+m w) .
\end{aligned}
$$

On the use of equations (4) and (5), the equations of motion along $x$-and $y$-directions are

$$
\begin{aligned}
2 \Omega w-v_{0} \frac{d u}{d y} & =-\frac{1}{\rho} \frac{\partial p}{\partial x}+v \frac{d^{2} u}{d z^{2}}-\frac{\sigma B_{0}}{\rho\left(1+m^{2}\right)}(u+m w), \\
-2 \Omega u-v_{0} \frac{d w}{d y} & =-\frac{1}{\rho} \frac{\partial p}{\partial z}+v \frac{d^{2} w}{d z^{2}}+\frac{\sigma B_{0}}{\rho\left(1+m^{2}\right)}(m u-w), \\
0 & =-\frac{1}{\rho} \frac{\partial p}{\partial y},
\end{aligned}
$$

where $\rho, v$ and $p$ are respectively the fluid density, the kinematic coefficient of viscosity and the modified fluid pressure including centrifugal force.

The boundary conditions are

$$
\begin{aligned}
& u=w=0 \quad \text { at } \quad z=0, \\
& u=U, w=0 \text { at } z=d .
\end{aligned}
$$

Under the usual boundary layer approximations, equations (6) and (7) become

$$
\begin{aligned}
2 \Omega w-v_{0} \frac{d u}{d y} & =-\frac{1}{\rho} \frac{\partial p}{\partial x}+v \frac{d^{2} u}{d z^{2}} \\
- & \frac{\sigma B_{0}^{2}}{\rho\left(1+m^{2}\right)}[(u-U)+m w], \\
-2 \Omega(u-U)-v_{0} \frac{d w}{d y} & =-\frac{1}{\rho} \frac{\partial p}{\partial x}+v \frac{d^{2} w}{d z^{2}} \\
& +\frac{\sigma B_{0}^{2}}{\rho\left(1+m^{2}\right)}[m(u-U)-w] .
\end{aligned}
$$

Introduce the non-dimensional variables

$$
\eta=\frac{z}{d}, u_{1}=\frac{u}{U}, w_{1}=\frac{w}{U},
$$

equations (10) and (11) become

$$
\begin{aligned}
& 2 K^{2} w_{1}-\operatorname{Re} \frac{d u_{1}}{d \eta}=\frac{d^{2} u_{1}}{d \eta^{2}}-\frac{M^{2}}{1+m^{2}}\left[\left(u_{1}-1\right)+m w_{1}\right], \\
& -2 K^{2}\left(u_{1}-1\right)-\operatorname{Re} \frac{d w_{1}}{d \eta}=\frac{d^{2} u_{1}}{d \eta^{2}}-\frac{M^{2}}{1+m^{2}}\left[m\left(u_{1}-1\right)-w_{1}\right],
\end{aligned}
$$

where $M^{2}=\frac{\sigma B_{0}^{2} d^{2}}{\rho v}$ is the Hartmann number, $K^{2}=\frac{\Omega d^{2}}{v}$ the rotation parameter and $R e=\frac{v_{0} U}{v}$ the Reynolds number.

Equations (13) and (14) can be combined into the following equation

$$
\frac{d^{2} F}{d \eta^{2}}+\operatorname{Re} \frac{d F}{d \eta}-\left[\frac{M^{2}(1-i m)}{1+m^{2}}-2 i K^{2}\right] F=0,
$$

where

$$
F=u_{1}+i w_{1}-1, i=\sqrt{-1}
$$

The boundary conditions for $F(\eta)$ are

$$
F=-1 \text { at } \eta=0 \text { and } F=0 \text { at } \eta=1 \text {. }
$$

The solution of the equation (15) subject to the boundary conditions (17) is

$$
F(\eta)=\left[\frac{\cosh (\alpha-i \beta)}{\sinh (\alpha-i \beta)} \sinh (\alpha-i \beta) \eta-\cosh (\alpha-i \beta) \eta\right] e^{-\frac{\mathrm{Re}}{2} \eta} .
$$

On the use of equation (16) and separating into real and imaginary parts, we get

$$
u_{1}=1-\frac{2 e^{-\frac{R e}{2} \eta}}{\cosh 2 \alpha-\cos 2 \beta}
$$

$\times[\sinh \alpha(1-\eta) \cos \beta(1-\eta) \sinh \alpha \cos \beta$ 
$+\cosh \alpha(1-\eta) \sin \beta(1-\eta) \cosh \alpha \sin \beta]$,

$w_{1}=-\frac{2 e^{-\frac{R e}{2} \eta}}{\cosh 2 \alpha-\cos 2 \beta}$

$\times[\sinh \alpha(1-\eta) \cos \beta(1-\eta) \cosh \alpha \sin \beta$

$-\cosh \alpha(1-\eta) \sin \beta(1-\eta) \sinh \alpha \cos \beta$ ],

where

$$
\begin{aligned}
& \alpha=\frac{1}{\sqrt{2}}\left[\left\{\left(\frac{R e^{2}}{4}+\frac{M^{2}}{1+m^{2}}\right)^{2}+\left(2 K^{2}+\frac{m M^{2}}{1+m^{2}}\right)^{2}\right\}^{\frac{1}{2}}\right. \\
& \left.+\left(\frac{R e^{2}}{4}+\frac{M^{2}}{1+m^{2}}\right)\right]^{\frac{1}{2}} \\
& \beta=\frac{1}{\sqrt{2}}\left[\left\{\left(\frac{R e^{2}}{4}+\frac{M^{2}}{1+m^{2}}\right)^{2}+\left(2 K^{2}+\frac{m M^{2}}{1+m^{2}}\right)^{2}\right\}^{\frac{1}{2}}\right. \\
& \left.-\left(\frac{R e^{2}}{4}+\frac{M^{2}}{1+m^{2}}\right)\right]^{\frac{1}{2}} .
\end{aligned}
$$

The solution given by equations (19) and (20) exists for both $R e<0$ (corresponding to $v_{0}<0$ for the blowing at the plates) and $R e>0$ (corresponding to $v_{0}>0$ for the suction at the plates). If $R e=0$ and $M^{2}=0$, then the above equations (19) and (20) are identical with equations (8) and (9) of Jana and Datta[10].

\section{RESULTS AND DISCUSSION}

To study the effects of rotation, suction/blowing and Hall parameter on the velocity distributions we have presented the non-dimensional velocity components $u_{1}$ and $w_{1}$ against $\eta$ in Figures.2-5 for various values of Hall parameter $m$, magnetic parameter $M^{2}$, the rotation parameter $K^{2}$ and Reynolds number $R e$. It is seen from Figure. 2 that the primary velocity $u_{1}$ increases with increase in magnetic parameter $M^{2}$ whereas the magnitude of the secondary velocity $w_{1}$ decreases with increase in magnetic parameter $M^{2}$. The retardation in the secondary velocity field due to the increase in the magnetic parameter is noticed form this figure. This observation can be explained by the following fact. As the magnetic parameter $M^{2}$ increases, the Lorentz force which opposes the flow, also increases and leads to the enhanced deceleration of the flow. Figure. 3 shows that the primary velocity $u_{1}$ decreases whereas the magnitude of the secondary velocity $w_{1}$ increases with increase in Hall parameter $m$. Hall parameter accelerates the secondary velocity field. Figure. 4 reveals that the primary velocity $u_{1}$ increases with increase in $K^{2}$. On the other hand, the magnitude of the secondary velocity $w_{1}$ increases near the stationary plate while it decreases near the moving plate with increase in rotation parameter $K^{2}$. The rotation parameter $K^{2}$ defines the relative magnitude of the Coriolis force and the viscous force in the regime, therefore it is clear that high magnitude Coriolis forces are counter-productive for the primary flow. It is observed from Figure.5 that the primary velocity $u_{1}$ increases while the magnitude of the secondary velocity $w_{1}$ decreases with increase in Reynolds number $R e$.

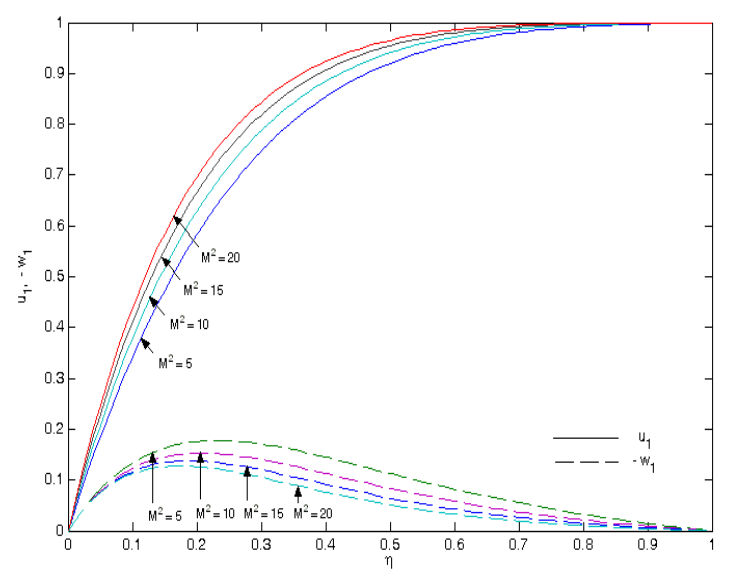

Figure 2. Velocity profiles for $M^{2}$ with $K^{2}=3, m=2$ and $\boldsymbol{R e}=\mathbf{2}$.

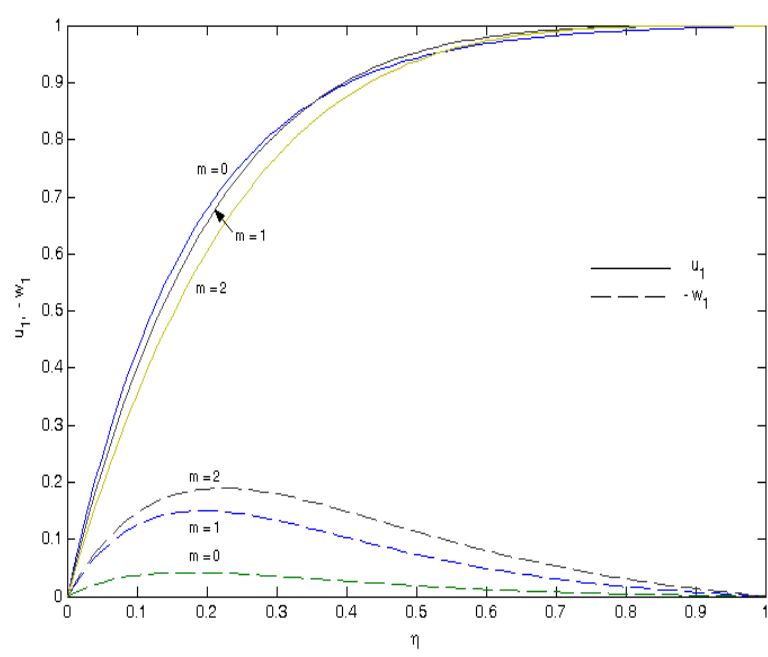

Figure 3. Velocity profiles for $m$ with $M^{2}=10, m=2$ and $\boldsymbol{R e}=\mathbf{2}$

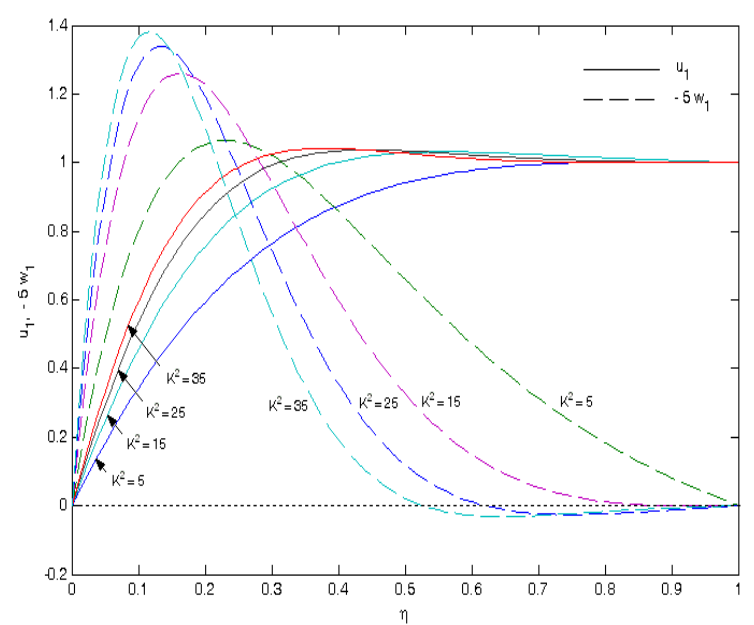

Figure 4. Velocity profiles for $K^{2}$ with $M^{2}=10, m=2$ and $\operatorname{Re}=\mathbf{2}$. 


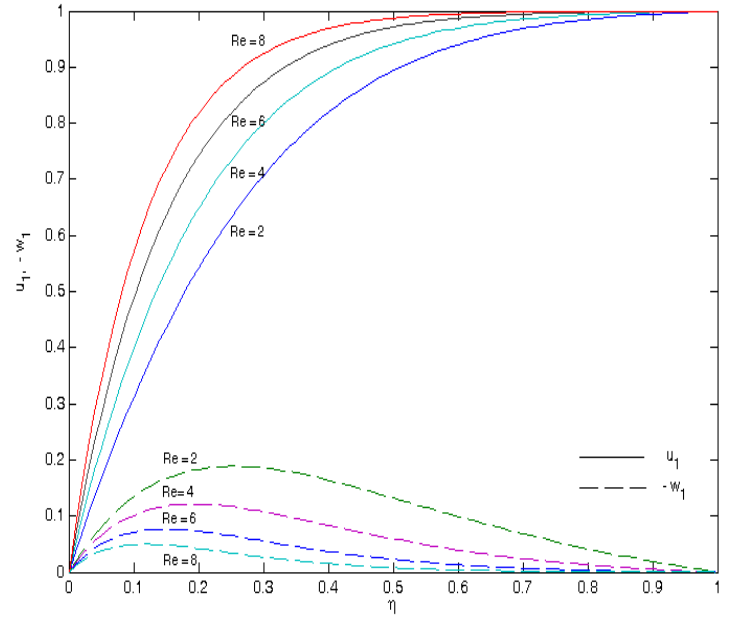

Figure 5. Velocity profiles for $R e$ with $M^{2}=10, K^{2}=3$ and $\boldsymbol{m}=\mathbf{2}$

The resultants of the non-dimensional shear stresses due to the primary and secondary velocities at the plates $\eta=0$ and $\eta=1$ are respectively given by

$$
\begin{aligned}
\tau_{0}= & {\left[\left(\frac{d u_{1}}{d \eta}\right)^{2}+\left(\frac{d w_{1}}{d \eta}\right)^{2}\right]_{\eta=0}^{\frac{1}{2}} } \\
= & {\left[\left\{\frac{1}{2} \operatorname{Re}+\frac{\alpha \sinh 2 \alpha+\beta \sin 2 \beta}{\cosh 2 \alpha-\cos 2 \beta}\right\}^{2}\right.} \\
& \left.+\left\{\frac{\alpha \sin 2 \beta-\beta \sinh 2 \alpha}{\cosh 2 \alpha-\cos 2 \beta}\right\}^{2}\right]^{\frac{1}{2}} \\
\tau_{1}=\left[\left(\frac{d u_{1}}{d \eta}\right)^{2}+\left(\frac{d w_{1}}{d \eta}\right)^{2}\right]_{\eta=1}^{\frac{1}{2}} & \frac{4 e^{-R e}}{\cosh 2 \alpha-\cos 2 \beta}\left[\left(\alpha^{2}+\beta^{2}\right)\left(\sinh ^{2} \alpha+\sin ^{2} \beta\right)\right]^{\frac{1}{2}},
\end{aligned}
$$

where $\alpha$ and $\beta$ are given by (21).

Numerical results of the resultant non-dimensional shear stresses $\tau_{0}$ and $\tau_{1}$ due to the primary and secondary flows at the plates $\eta=0$ and $\eta=1$ are shown graphically in Figures. 68 against $M^{2}$ for different values of $m, K^{2}$ and $R e$. Figure.6 shows that the resultant shear stress $\tau_{0}$ due to the primary flow at the stationary plate $\eta=0$ decreases with increase in Hall parameter $m$ for fixed value of $M^{2}$ while it increases with increase in magnetic parameter $M^{2}$. Further, it is seen that the resultant shear stress $\tau_{1}$ due to the secondary flow at the moving plate $\eta=1$, decreases with increase in either $m$ or $M^{2}$. Since the primary velocity increases with an increase in $M^{2}$, the shear stess $\tau_{0}$ also increases with $M^{2}$, because the friction increases with the fluid velocity. Similarly, the secondary velocity decreases as $M^{2}$ increases. Therefore, the shear stess $\tau_{0}$ also exhibits a similar behaviour as $M^{2}$ increases. It is observed from Figure.7 that the shear stess $\tau_{0}$ increases with increase in either rotation parameter $K^{2}$ or magnetic parameter $M^{2}$ or Reynolds number $R e$. Figure.8 reveals that the shear stess $\tau_{1}$ decreases with increase in either rotation parameter $K^{2}$ or Reynolds number $R e$. Due to the increase in parameter $K^{2}$ or $R e$, the secondary velocity decreases. It is also seen that for $K^{2} \leq 4$ or $\operatorname{Re} \leq 5, \tau_{1}$ first decreases, reaches a minimum and then it increases in magnetic parameter $M^{2}$ while it decreases with increase in $M^{2}$ for $K^{2}>4$ or $R e>5$.

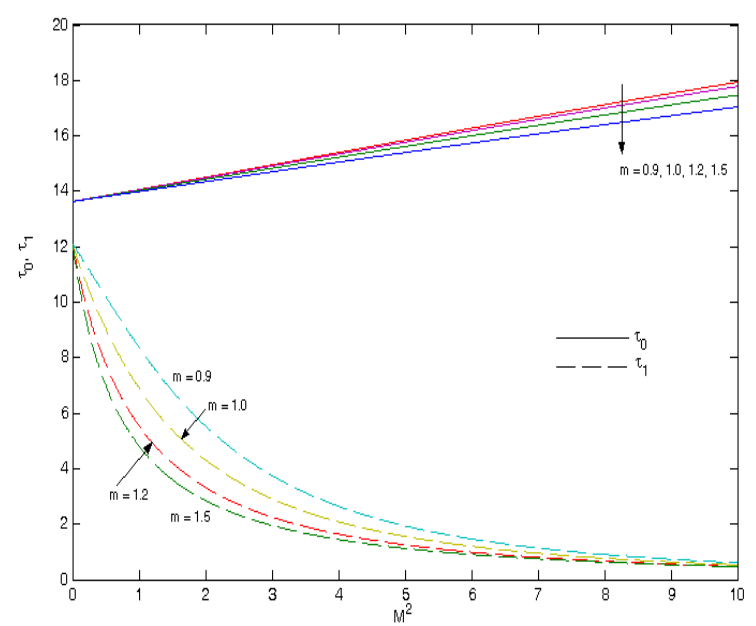

Figure 6. Shear stresses for $m$ with $K^{2}=3$ and $R e=2$

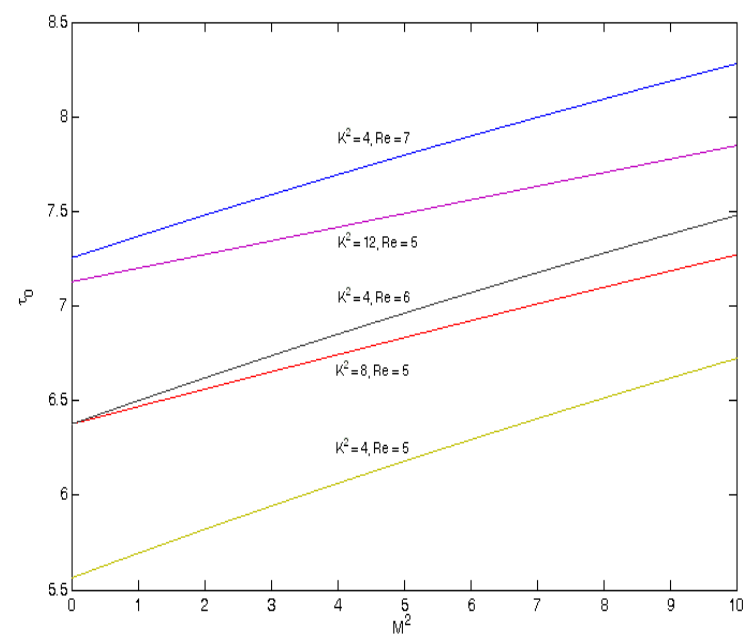

Figure 7. Shear stress due to primary flow for both $K^{2}$ and $R e$ with $m=0.5$ 


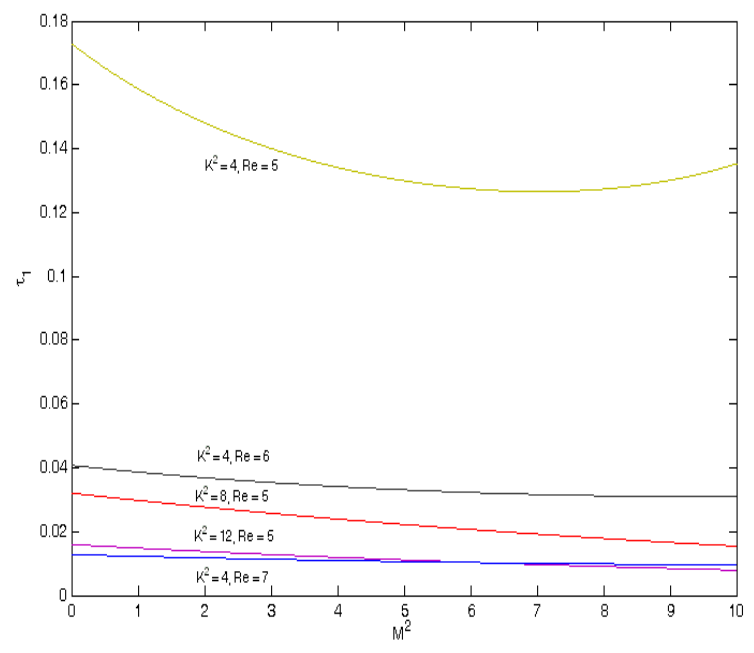

Figure 8. Shear stress due to secondary flow for both $K^{2}$ and $R e$ with $m=2$

Asymptotic behavior of the solutions (19) and (20) are analyzed for large values of $M^{2}, K^{2}$ and $R e$.

Case (i): When $K^{2}=1, M^{2}=1$ and $\operatorname{Re}=1$.

In this case, equations (19) and (20) yield

$$
\begin{aligned}
& u_{1}(\eta)=\eta+\frac{1}{2} \operatorname{Re} \eta(1-\eta)+\frac{1}{6} \cdot \frac{M^{2}}{1+m^{2}} \eta(1-\eta)(2-\eta), \\
& w_{1}(\eta)=-\left(2 K^{2}+\frac{m M^{2}}{1+m^{2}}\right) \eta(1-\eta)(2-\eta) .
\end{aligned}
$$

It is seen from equations (24) and (25) that for small values of $K^{2}, M^{2}$ and $R e$, the primary velocity is independent of the rotation parameter $K^{2}$. On the other hand, the secondary velocity depends on the rotation parameter as well as magnetic and Hall parameters while it is independent of the Reynolds number. Further, if $K^{2}=0=M^{2}=R e$, then equations (25) and (25) reduced to $u_{1}(\eta)=\eta$ and $w_{1}(\eta)=0$ which represent the velocity components in the plane Couette flow.

Case (ii): When $K^{2} ? 1, M^{2}=1$ and $R e=1$. In this case, equations (19) and (20) become

$$
\begin{aligned}
& u_{1}=1-e^{-\left(\frac{R e}{2}+\alpha\right) \eta} \cos \beta \eta, \\
& w_{1}=-e^{-\left(\frac{R e}{2}+\alpha\right) \eta} \sin \beta \eta,
\end{aligned}
$$

where

$$
\begin{aligned}
& \alpha=K\left[1+\frac{1}{4 K^{2}}\left\{\frac{R e^{2}}{4}+\frac{(1+m) M^{2}}{1+m^{2}}\right\},\right. \\
& \beta=K\left[1-\frac{1}{4 K^{2}}\left\{\frac{R e^{2}}{4}+\frac{(1-m) M^{2}}{1+m^{2}}\right\} .\right.
\end{aligned}
$$

It is seen from equations (26) and (27) that there exists a single-deck boundary layer of thickness of the order $\mathrm{O}\left(\frac{R e}{2}+\alpha\right)^{-1}$ where $\alpha$ is given by (28). It is seen that the thickness of this boundary layer increases with increase in Hall parameter $m$ but it decreases with increase in either Hartmann number $M$ or $R e$ or $K^{2}$.

Case (iii) : When $M^{2} ? 1, K^{2}=1$ and $R e=1$.

In this case, the velocity distributions are obtained from equations (19) and (20) as

$$
\begin{aligned}
& u_{1}=1-e^{-\left(\frac{R e}{2}+\alpha\right) \eta} \cos \beta \eta, \\
& w_{1}=e^{-\left(\frac{R e}{2}+\alpha\right) \eta} \sin \beta \eta,
\end{aligned}
$$

where

$$
\alpha=\left(\frac{M^{2}}{1+m^{2}}\right)^{\frac{1}{2}}, \beta=\frac{1}{2}\left(2 K^{2}+\frac{m M^{2}}{1+m^{2}}\right)\left(\frac{1+m^{2}}{M^{2}}\right)^{\frac{1}{2}} .
$$

Equations (29) and (30) show the existence of single-decker boundary layer of thickness of the order $\mathrm{O}\left(\frac{R e}{2}+\alpha\right)^{-1}$. This thickness of the layer increases with increase in Hall parameter $m$ while it decreases with increase in either $R e$ or $M^{2}$. It is interesting to note that for large values of $M^{2}$, the boundary layer thickness is independent of rotation parameter.

Case (iv): When $R e$ ? $1, M^{2}=1$ and $K^{2}=1$.

In this case, the velocity distributions given by (19) and (20) become

$$
\begin{aligned}
& u_{1}=1-e^{-R e \eta} \cos \beta \eta, \\
& w_{1}=e^{-R e \eta} \sin \beta \eta,
\end{aligned}
$$

where

$$
\alpha=\frac{\operatorname{Re}}{2}, \beta=\frac{1}{\operatorname{Re}}\left(2 K^{2}+\frac{m M^{2}}{1+m^{2}}\right) .
$$

Equations (32) and (33) show the existence of single-deck boundary layer of thickness of this boundary layer the order $\mathrm{O}\left(R e^{-1}\right)$. The thickness of the layer decreases with increase in Reynolds number $R e$. It is noticed that for large values of the Reynolds number $R e$, the boundary layer thickness is independent of Hall parameter, magnetic parameter as well as rotation parameter.

\section{HEAT TRANSFER}

The energy equation for the fully developed flow including viscous and Joule dissipation is

$$
\begin{aligned}
-\rho C_{p} v_{0} \frac{d T}{d y} & =k \frac{d^{2} T}{d y^{2}}+\mu\left[\left(\frac{d u}{d y}\right)^{2}+\left(\frac{d w}{d y}\right)^{2}\right] \\
& +\frac{1}{\sigma}\left(j_{x}^{2}+j_{y}^{2}\right),
\end{aligned}
$$

where $C_{p}$ is the specific heat at constant pressure and $k$ is the thermal conductivity.

The temperature boundary conditions are

$$
T=T_{0} \text { at } y=0, T=T_{1} \text { at } y=d,
$$

where $T_{0}$ and $T_{1}\left(T_{1}>T_{0}\right)$ denote the uniform temperature of the plates at $y=0$ and $y=d$, respectively.

Introducing 


$$
\theta=\frac{T-T_{0}}{T_{1}-T_{0}},
$$

and on the use of (12) and (16), the energy equation (37) can be written in a dimensionless form as

$$
\frac{d^{2} \theta}{d \eta^{2}}+\operatorname{Pr} E c \frac{d \theta}{d \eta}=\operatorname{Pr} E c\left(\frac{d F}{d \eta} \frac{d \bar{F}}{d \eta}+\frac{M^{2}}{1+m^{2}} F \bar{F}\right),
$$

where $\operatorname{Pr}=\frac{\mu C_{p}}{k}$ is the Prandtl number and $E c=\frac{U^{2}}{C_{p}\left(T_{1}-T_{0}\right)}$ the Eckert number.

The corresponding temperature boundary conditions for $\theta(\eta)$ become

$$
\theta(0)=0 \text { and } \theta(1)=1 .
$$

Using equations (19) and (20), the solution of equation (38) subject to the boundary conditions (39) is

$$
\theta(\eta)=\left\{\begin{array}{l}
d_{1}+d_{2} e^{-\operatorname{Pr} R e \eta}+\frac{\operatorname{Pr} E c e^{-\operatorname{Re} \eta}}{2(\cosh 2 \alpha-\cos 2 \beta)} \\
\times\left[\left(c_{1}+c_{2}\right) \cosh 2 \alpha(1-\eta)\right. \\
+\left(c_{1}-c_{2}\right) \sinh 2 \alpha(1-\eta)+c_{3} \cos 2 \beta(1-\eta) \\
\left.+c_{4} \sin 2 \beta(1-\eta)\right] \\
\text { for } \operatorname{Re}+2 \alpha \neq \operatorname{RePr} \text { and }-2 \alpha+\operatorname{Re} \neq \operatorname{RePr} r \\
\times\left[\left(c_{1}^{*} \eta+c_{2}\right) \cosh 2 \alpha(1-\eta)\right. \\
+\left(c_{1}^{*} \eta-c_{2}\right) \sinh 2 \alpha(1-\eta)+c_{3} \cos 2 \beta(1-\eta) \\
\left.+c_{4} \sin 2 \beta(1-\eta)\right], \\
\text { for } \operatorname{Re}+2 \alpha=\operatorname{Re} \operatorname{Pr} \text { and }-2 \alpha+\operatorname{Re} \neq \operatorname{RePr} \\
d_{1}+d_{2} e^{-\operatorname{Pr} \operatorname{Re} \eta}+\frac{\operatorname{Pr} E c e^{-\operatorname{Re} \eta}}{2 \alpha-\cos 2 \beta)} \\
d_{1}+d_{2} e^{-\operatorname{Pr} \operatorname{Re} \eta}+\frac{\operatorname{Pr} E c e^{-\operatorname{Re} \eta}}{2(\cosh 2 \alpha-\cos 2 \beta)} \\
\times\left[\left(c_{1}+c_{2}^{*} \eta\right) \cosh 2 \alpha(1-\eta)\right. \\
+\left(c_{1}-c_{2}^{*} \eta\right) \sinh 2 \alpha(1-\eta)+c_{3} \cos 2 \beta(1-\eta) \\
\left.+c_{4} \sin 2 \beta(1-\eta)\right], \\
\text { for } \operatorname{Re}+2 \alpha \neq \operatorname{RePr} \text { and }-2 \alpha+\operatorname{Re}=\operatorname{RePr}
\end{array}\right.
$$

where

$$
\begin{aligned}
& c_{1}=\frac{\left(\frac{R e}{2}+\alpha\right)^{2}+\beta^{2}+\frac{M^{2}}{1+m^{2}}}{(\operatorname{Re}+2 \alpha)(\operatorname{Re}+2 \alpha-\operatorname{RePr})}, \\
& c_{1}^{*}=-\frac{\left(\frac{\operatorname{Re}}{2}+\alpha\right)^{2}+\beta^{2}+\frac{M^{2}}{1+m^{2}}}{(\operatorname{Re}+2 \alpha)}, \\
& c_{2}=\frac{\left(-\frac{\operatorname{Re}}{2}+\alpha\right)^{2}+\beta^{2}+\frac{M^{2}}{1+m^{2}}}{(-\operatorname{Re}+2 \alpha)(-\operatorname{Re}+2 \alpha-\operatorname{RePr})}, \\
& c_{2}^{*}=-\frac{\left(-\frac{\operatorname{Re}}{2}+\alpha\right)^{2}+\beta^{2}+\frac{M^{2}}{1+m^{2}}}{(-2 \alpha+\operatorname{Re})} \\
& c_{3}=\frac{2\left(A_{1} A_{2}+B_{1} B_{2}\right)}{A_{2}^{2}+B_{2}^{2}}, c_{4}=\frac{2\left(B_{1} B_{2}-A_{1} A_{2}\right)}{A_{2}^{2}+B_{2}^{2}},
\end{aligned}
$$

$$
\begin{aligned}
& A_{1}=\alpha^{2}+\beta^{2}-\frac{R e^{2}}{4}-\frac{M^{2}}{1+m^{2}}, B_{1}=\beta R e, \\
& A_{2}=\operatorname{Re}^{2}-4 \beta^{2}-\operatorname{Re}^{2} \operatorname{Pr}, B_{2}=2 \beta \operatorname{Re}(2-\operatorname{Pr}) .
\end{aligned}
$$

The constant $d_{1}$ and $d_{2}$ in the equations (40) are obtained on using the conditions (39). The effect of the Hall parameter $m$, rotation parameter $K^{2}$ and Reynolds number $R e$ on the temperature distribution has been shown in Figures.9-11. It is seen from Figure.9 that the temperature increases near the stationary plate $\eta=0$ and closeness near the moving plate $\eta=1$ with increase in the Hall parameter $m$. Thus, the Hall parameter $m$ accelerates the channel temperature. It is observed from Figure. 10 that the temperature decreases with increase in the rotation parameter $K^{2}$. The effect of the rotation parameter $K^{2}$ is to decrease the temperature in the channel. Because of rotation, it is seen that the rate of heat transfer at the upper plate decreases. Increasing rotation therefore opposes the conduction of heat from the upper plate of the channel into the fluid, causing a decrease in fluid temperature in the channel. Figure.11 shows that with increase in the Reynolds number the temperature of fluid increases.

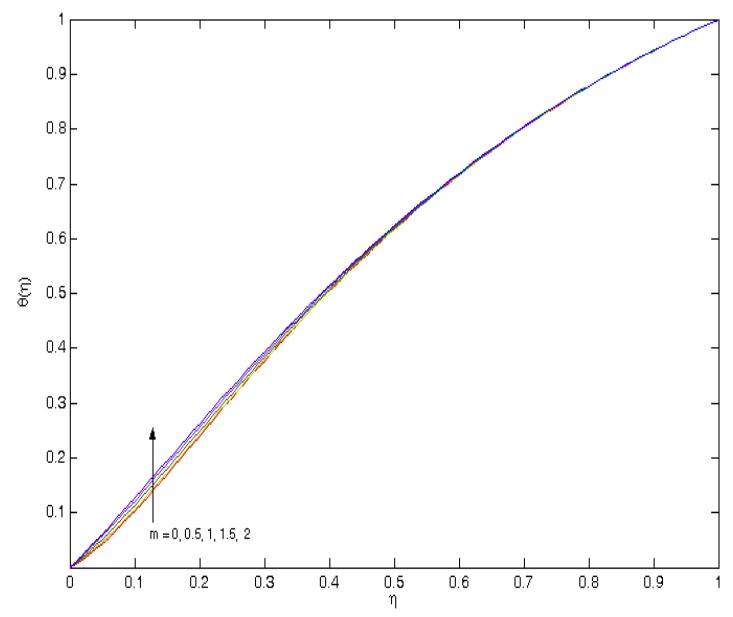

Figure 9. Temperature profiles for $m$ with $M^{2}=10$, $K^{2}=3$ and $R e=2$

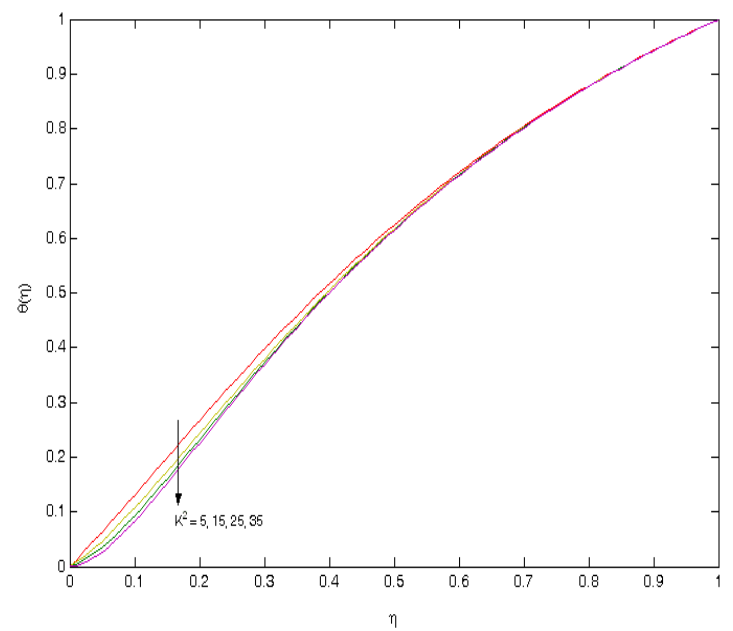

Figure 10. Temperature profiles for $K^{2}$ with $M^{2}=10$, $m=2$ and $R e=2$. 


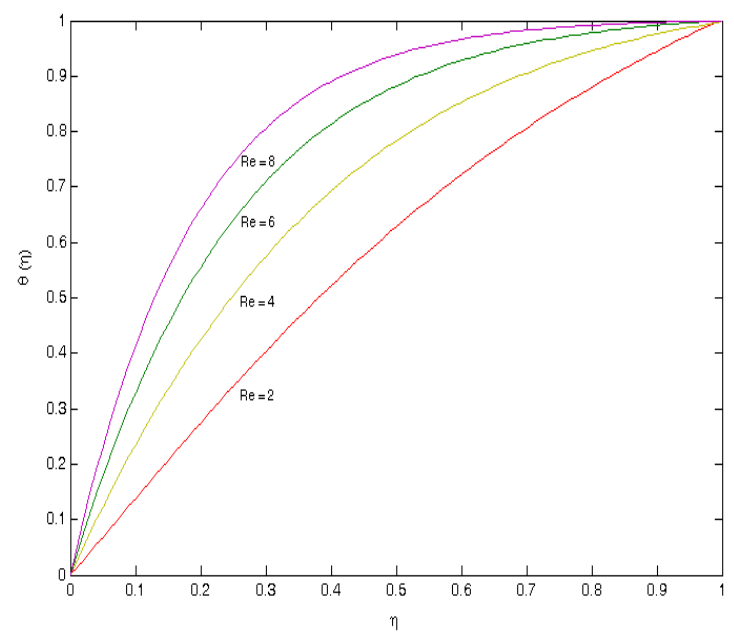

Figure 11. Temperature profiles for $R e$ with $M^{2}=10$, $K^{2}=3$ and $m=2$.

The rate of heat transfer at the plates $\eta=0$ and $\eta=1$ can be obtained from (40) as

$$
\theta^{\prime}(0)=\left\{\begin{array}{l}
-\operatorname{RePr} d_{2}-\frac{\operatorname{Pr} E c}{2(\cosh 2 \alpha-\cos 2 \beta)} \\
\times\left[\left\{R_{e}\left(c_{1}+c_{2}\right)+2 \alpha\left(c_{1}-c_{2}\right)\right\} \cosh 2 \alpha\right. \\
+\left\{2 \alpha\left(c_{1}+c_{2}\right)+\operatorname{Re}\left(c_{1}-c_{2}\right)\right\} \sinh 2 \alpha \\
+\left(R_{e} c_{3}+2 c_{4} \beta\right) \cos 2 \beta \\
\left.+\left(\operatorname{Rec} c_{4}-2 c_{3} \sin 2 \beta\right)\right] \\
\text { for } R_{e}+2 \alpha \neq \operatorname{RePr} \text { and }-2 \alpha+\operatorname{Re} \neq \operatorname{RePr} \\
\times\left[c_{1}^{*}(\cosh 2 \alpha+\sinh 2 \alpha)\right. \\
+(2 \alpha-\operatorname{Re}) c_{2}(\cosh 2 \alpha-\sinh 2 \alpha) \\
+\left(2 \beta c_{3}-\operatorname{Rec} c_{4}\right) \sin 2 \beta \\
\left.-\left(2 \beta c_{4}-\operatorname{Rec} c_{3}\right) \cos 2 \beta\right] \\
\text { for } \operatorname{Re}+2 \alpha=\operatorname{Re} \operatorname{Pr} \text { and }-2 \alpha+\operatorname{Re} \neq \operatorname{RePr} \\
\\
-\operatorname{Rer} d_{2}+\frac{\operatorname{Pr} E c}{2(\cosh 2 \alpha-\cos 2 \beta)} \\
\times\left[c_{2}^{*}(\cosh 2 \alpha-\sinh 2 \alpha)\right. \\
-(2 \alpha+\operatorname{Re}) c_{1}(\cosh 2 \alpha+\sinh 2 \alpha) \\
+\left(2 \beta c_{3}-\operatorname{Rec} c_{4}\right) \sin 2 \beta \\
\left.-\left(2 \beta c_{4}+\operatorname{Rec} c_{3}\right) \cos 2 \beta\right] \\
\text { for } \operatorname{Re}+2 \alpha \neq \operatorname{Re} \operatorname{Pr} \text { and }-2 \alpha+\operatorname{Re}=\operatorname{RePr},
\end{array}\right.
$$

$$
\theta^{\prime}(1)=\left\{\begin{array}{l}
-\operatorname{RePr} d_{2}-\frac{\operatorname{Pr} E c}{2(\cosh 2 \alpha-\cos 2 \beta)} \\
\times\left[\operatorname{Re}\left(c_{1}+c_{2}\right)+2 \alpha\left(c_{1}-c_{2}\right)+\operatorname{Re} c_{3}+2 c_{4} \beta\right] \\
\text { for } \operatorname{Re}+2 \alpha \neq \operatorname{Re} \operatorname{Pr} \text { and }-2 \alpha+\operatorname{Re} \neq \operatorname{RePr} \\
-\operatorname{Re} \operatorname{Pr} d_{2} e^{-\operatorname{PrRe}}+\frac{\operatorname{PrEc}}{2(\cosh 2 \alpha-\cos 2 \beta)} \\
\times\left[c_{1}^{*}-2 \alpha\left(c_{1}^{*}-c_{2}\right)-2 \beta c_{4}-\operatorname{Re}\left(c_{1}+c_{2}^{*}+c_{3}\right)\right] \\
\text { for } \operatorname{Re}+2 \alpha=\operatorname{Re} \operatorname{Pr} \text { and }-2 \alpha+\operatorname{Re} \neq \operatorname{RePr} \\
-\operatorname{RePr} d_{2} e^{-\operatorname{PrRe}}+\frac{\operatorname{PrEc}}{2(\cosh 2 \alpha-\cos 2 \beta)} \\
\times\left[c_{2}^{*}-2 \alpha\left(c_{1}-c_{2}^{*}\right)-2 \beta c_{4}-\operatorname{Re}\left(c_{1}+c_{2}^{*}+c_{3}\right)\right] \\
\text { for } \operatorname{Re}+2 \alpha \neq \operatorname{Re} \operatorname{Pr} \text { and }-2 \alpha+\operatorname{Re}=\operatorname{RePr}
\end{array}\right.
$$


Table 1. Rate of heat transfer at the plates $\eta=0$ and $\eta=1$ with $R e=2$ and $K^{2}=5$.

\begin{tabular}{|c|c|c|c|c|c|c|c|c|}
\hline & \multicolumn{5}{|c|}{$\theta^{\prime}(0)$} & \multicolumn{5}{c|}{$\theta^{\prime}(1)$} \\
\hline$m \backslash M^{2}$ & 5 & 10 & 15 & 20 & 5 & 10 & 15 & 20 \\
\hline 0.0 & 1.04694 & 0.82908 & 0.62614 & 0.44045 & 0.51481 & 0.51723 & 0.51919 & 0.52062 \\
0.5 & 1.03751 & 0.83453 & 0.65270 & 0.48785 & 0.51530 & 0.51740 & 0.51906 & 0.52033 \\
1.0 & 1.08313 & 0.92293 & 0.77919 & 0.64811 & 0.51500 & 0.51660 & 0.51799 & 0.51913 \\
1.5 & 1.12624 & 1.00211 & 0.88940 & 0.78568 & 0.51467 & 0.51584 & 0.51695 & 0.51793 \\
\hline
\end{tabular}

Table 2 . Rate of heat transfer at the plates $\eta=0$ and $\eta=1$ with $M^{2}=5$ and $K^{2}=5$.

\begin{tabular}{|c|c|c|c|c|c|c|c|c|}
\hline & \multicolumn{5}{|c|}{$\theta^{\prime}(0)$} & \multicolumn{4}{c|}{$\theta^{\prime}(1)$} \\
\hline$m \backslash R e$ & 2 & 4 & 6 & 8 & 2 & 4 & 6 & 8 \\
\hline 0.0 & 1.04694 & 2.17003 & 3.40052 & 4.65976 & 0.51481 & 0.19506 & 0.06688 & 0.02118 \\
0.5 & 1.03751 & 2.16970 & 3.41174 & 4.67711 & 0.51530 & 0.19514 & 0.06684 & 0.02117 \\
1.0 & 1.08313 & 2.21705 & 3.46126 & 4.72416 & 0.51500 & 0.19506 & 0.06670 & 0.02113 \\
1.5 & 1.12624 & 2.25921 & 3.50182 & 4.76017 & 0.51467 & 0.19474 & 0.06657 & 0.02110 \\
\hline
\end{tabular}

The numerical values of the rate of heat transfers $\theta^{\prime}(0)$ and $\theta^{\prime}(1)$ are entered in the Tables 1 and 2 for different values of $M^{2}, \operatorname{Re}$ and $m$ with $K^{2}=5$. It is seen from the Table 1 that for $M^{2}>5$, the rate of heat transfer $\theta^{\prime}(0)$ at the stationary plate $\eta=0$ increases with increase in Hall parameter $m$ while for $M^{2} \leq 5$, the rate of heat transfer decreases and reaches a minimum and then increases with increase in $m$. On the other hand, for fixed value of $m$, the rate of heat transfer at the plate $\eta=0$ decreases with increase in $M^{2}$. It is observed from Table 2 that the rate of heat transfer $\theta^{\prime}(0)$ increases with increase in Reynolds number $R e$. Tables 1 and 2 show that with increase in $m$ the rate of heat transfer $\theta^{\prime}(1)$ at the plate $\eta=1$, decreases either for $M^{2} \leq 10$ or $R e \leq 4$ while it first decreases and reaches a minimum and then increases either for $M^{2}>10$ or $R e>4$ with increase in $m$.

\section{SINGLE OSCILLATING PLATE}

In the limit $d \rightarrow \infty$, when the moving plate is at an infinite distance, the solution (18) becomes, on using (16)

$$
\begin{aligned}
& u_{1}=1-e^{-\left(\frac{S}{2}+\alpha\right) \eta} \cos \beta \eta, \\
& w_{1}=-e^{-\left(\frac{S}{2}+\alpha\right) \eta} \sin \beta \eta,
\end{aligned}
$$

where

$$
\begin{gathered}
\alpha=\frac{1}{\sqrt{2}}\left[\left\{\left(\frac{S^{2}}{4}+\frac{M^{2}}{1+m^{2}}\right)^{2}+\left(2 K^{2}+\frac{m M^{2}}{1+m^{2}}\right)^{2}\right\}^{\frac{1}{2}}\right. \\
\left.+\left(\frac{S^{2}}{4}+\frac{M^{2}}{1+m^{2}}\right)\right]^{\frac{1}{2}} \\
\beta=\frac{1}{\sqrt{2}}\left[\left\{\left(\frac{S^{2}}{4}+\frac{M^{2}}{1+m^{2}}\right)^{2}+\left(2 K^{2}+\frac{m M^{2}}{1+m^{2}}\right)^{2}\right\}^{\frac{1}{2}}\right.
\end{gathered}
$$

$$
\begin{gathered}
\left.-\left(\frac{S^{2}}{4}+\frac{M^{2}}{1+m^{2}}\right)\right]^{\frac{1}{2}}, \\
S=\frac{v_{0}}{U}, M^{2}=\frac{\sigma \mu_{e}^{2} H_{0}^{2} v}{\rho U^{2}}, K^{2}=\frac{\Omega v}{U^{2}} .
\end{gathered}
$$

Equations (44) and (45) are the velocity components due to free stream in the presence of a uniform transverse magnetic field and Hall currents when both the plate and the fluid rotate in unison with constant angular velocity $\Omega$ about an axis normal to the plate. The above expressions (44) and (45) show the existence of single-decker boundary layer of thicknesses of the order $\mathrm{O}\left(\frac{S}{2}+\alpha\right)^{-1}$. The thickness of the layer increases with increase in Hall parameter $m$ while it decreases with increase in either $K^{2}$ or $M^{2}$. In the absence of the rotation, equations (44) and (45) identical with the equations (8) and (9) of Pop and Soundalgekar [11] and the equations (37) and (38) of Gupta [12]. Further, if $M=0$ and $m=0$, then the equations (44) and (45) are identical with the results obtained by Gupta [13].

As $d \rightarrow \infty$, the temperature distribution given by (40) becomes

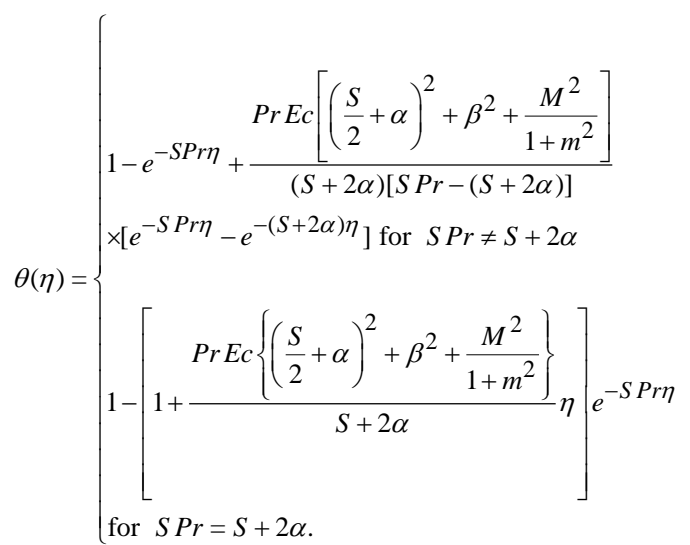

Equation (47) is identical with the equations (31) and (34) of Mazumder et al. [14] when Joule dissipation is neglected. It is seen from the above equations that the thermal boundary layer 
has a double-deck structure of thicknesses of the order $\mathrm{O}(1 / S P r)$ and $\mathrm{O}\left\{(S+2 \alpha)^{-1}\right\}$ when $\operatorname{SPr} \neq 2 \alpha$ while for $S P r=2 \alpha$ it reduces to single-deck structure of layer of order $\mathrm{O}(1 / S P r)$. It is evident from the expression of $\alpha$ given by (46) that the thickness of thermal boundary layer increases with increase in Hall parameter $m$ while it decreases with increase in either $K^{2}$ or $M^{2}$ or $\operatorname{Re}$. This is due to the fact that $\alpha$ decreases with increase in Hall parameter $m$ and it increases with increase in either $K^{2}$ or $M^{2}$ or Re.

\section{CONCLUSION}

Hall effect on MHD Couette flow between infinite horizontal parallel plates in a rotating system under boundary layer approximation has been studied. For large values of rotation parameter $K^{2}$, magnetic parameter $M^{2}$ and Reynolds number $\mathrm{Re}$, there exists a single-deck boundary layer near the plate $\eta=0$. Further, when the moving plate is placed at infinity $(d \rightarrow \infty)$ then we arrive at the problem of the conducting fluid past on infinite porous flat plate with free-stream velocity $U$. The thickness of the boundary layer increases with increases in Hall parameter either for large values of rotation or magnetic parameter while for large values of Reynolds number it is independent of Hall parameter.

\section{REFERENCES}

[1] Gupta, A. S.(1972). Heat transfer in hydromagnetic couette flow with Hall effects. The Mathematics Student. XL: 103-106.

[2] Jana, R. N., Datta, N. and Mazumder, B. S.(1977). Magnetohydrodynamic Couette flow and heat transfer in a rotating system. J. Phys. Soc. Japn. 42:1034-1039.

[3] Soundalgekar, V. M., Dessai, G. A. and Gupta, A. S.(1974). Hall effects on generalized MHD Couete flow with heat transfer. Bulletin De La Classe Des Sciences, Lx: 332-345.
[4] Ghosh, S. K. and Pop, I. (2004). Hall effects on MHD plasma Couette flow in a rotating environment. Int. J. Applied Mech. Eng. 9(2): 293-305.

[5] Ghosh, S. K. (2002). Effects of Hall current on MHD Couette flow in a rotating system with arbitrary magnetic field. Czech. J. Phys. 52(1): 51-63.

[6] Jana, R. N. and Datta, N.(1977). Hall effects on unsteady Couette flow, Int. J. Engng. Sci.15:35-43.

[7] Kanch, A. K. and Jana, R. N.(2001). Hall effect on unsteady Couette flow under boundary layer approximations. J. Physical Sciences. 7: 74-86.

[8] Cowling T. G. (1957). Magnetohydrodynamics, New york, Intersscience, pp.101.

[9] Batchelor, G. K. (1967). An Introduction to Fluid Dynamics, Ist ed., Cambridge Press, Cambridge, U. K., pp.200.

[10] Jana, R. N. and Datta, N.(1977). Couette flow and heat transfer in a rotating system, Acta Meccanica. 26: 301-306.

[11] Pop, I. and Soundalgekar, V. M.(1974). Effects of Hall currents on hyromagnetic flow near a porous plate. Acta Meccanica. 20:315-318.

[12] Gupta, A. S.(1975). Hydromagnetic flow past a porous plate with Hall currents, Acta Meccanica. 22: 281-287.

[13] Gupta, A. S.(1972). Ekman layer on a porous plate, Physics of fluids, 15: 930-931.

[14] Majumder, B. S., Gupta, A. S. and Datta, N.(1976). Flow and heat transfer in the hydromagnetic Ekman layer on a porous plate with Hall effects, Int. J. Heat and Mass transfer. 19: 523-527. 